\title{
ASYMPTOTIC ESTIMATES OF SUMS INVOLVING THE MOEBIUS FUNCTION. II
}

BY

\author{
KRISHNASWAMI ALLADI
}

\begin{abstract}
Let $n$ be a positive integer and $\mu(n)$ the Moebius function. If $n>1$, let $P(n)$ denote its largest prime factor and put $P(1)=1$. We study the asymptotic behavior of the sum $M^{*}(x, y)=\Sigma_{1<n<x, P(n)<y} \mu(n)$ as $x, y \rightarrow \infty$ and discuss a few applications.
\end{abstract}

1. Introduction. For a positive integer $n$ let $P(n)$ denote its largest prime factor if $n>1$, and put $P(1)=1$. Let $\mu(n)$ be the Moebius function. The aim of this paper is to study the asymptotic behavior of the sum

$$
M^{*}(x, y)=\sum_{\substack{1 \leqslant n \leqslant x \\ P(n)<y}} \mu(n)
$$

as $x, y \rightarrow \infty$, and discuss some applications.

In a recent paper [2], I studied the sum

$$
M(x, y)=\sum_{\substack{1 \leqslant n \leqslant x \\ p(n)>y}} \mu(n),
$$

where $p(n)$ is the smallest prime factor of $n$ if $n>1$ and $p(1)=\infty$. There are striking differences in the asymptotic behavior of these two functions and this motivated the present exposition.

The functions $M^{*}(x, y)$ and $M(x, y)$ can be considered as weighted versions of the two well-known functions

$$
\Psi(x, y)=\sum_{\substack{1 \leqslant n \leqslant x \\ P(n) \leqslant y}} 1
$$

and

$$
\Phi(x, y)=\sum_{\substack{1 \leqslant n \leqslant x \\ p(n) \geqslant y}} 1
$$

respectively. If $\alpha$ remains fixed as $x \rightarrow \infty$, it is known $($ see $[5,4])$ that

$$
\lim _{x \rightarrow \infty} \frac{\Psi\left(x, x^{1 / \alpha}\right)}{x}=\rho(\alpha)
$$

Received by the editors March 13, 1981; presented to the Society on November 15, 1980 (782nd AMS meeting).

1980 Mathematics Subject Classification. Primary 10H15, 10H25; Secondary 10A20.

Key words and phrases. Moebius function, asymptotic estimate, largest and smallest prime factors. 
and

$$
\lim _{x \rightarrow \infty} \frac{\Phi\left(x, x^{1 / \alpha}\right)}{(x / \log x)}=\alpha \cdot w(\alpha)
$$

both exist for every $\alpha>1$, and that $\rho(\alpha)$ and $w(\alpha)$ satisfy certain differencedifferential equations. By the use of analytic methods de Bruijn $[4,5]$ determined the asymptotic behavior of $\Phi(x, y)$ and $\Psi(x, y)$ as $x, y \rightarrow \infty$ independently. In [5] he expressed the opinion that the asymptotic behavior of $\Psi(x, y)$ is more complicated than $\Phi(x, y)$. Similarly we feel that $M^{*}(x, y)$ has a more complicated asymptotic behavior than $M(x, y)$. We now summarize our results.

Let $2 \leqslant y \leqslant x$ and $\beta=x / y$. We show in $\$ 2$ that for $y \geqslant \sqrt{x}$

$$
M^{*}(x, y)=\frac{x f(\beta)}{\log y}-\frac{x}{\log ^{2} x}\{1+O(R(\beta))\}+O\left(\frac{x}{\log ^{3} x}\right),
$$

where $f(\beta)$ and $R(\beta)$ are continuous functions that tend rapidly to zero as $\beta \rightarrow \infty$.

Next, let $\alpha=\log x / \log y$, where $2 \leqslant y \leqslant x$. If $\alpha>2$, we show in $\S 3$ that

$$
M^{*}(x, y)=\frac{\dot{x m^{*}}(\alpha)}{\log ^{2} y}+O_{\alpha}\left(\frac{x}{\log ^{3} y}\right),
$$

where $m^{*}(\alpha)$ is continuous for $\alpha>2$ and is given by means of a difference-differential equation. From (1.7) and (1.8) it easily follows that if $\alpha$ is fixed, then

$$
\lim _{x \rightarrow \infty} \frac{M^{*}(x, y)}{\left(x / \log ^{2} y\right)}
$$

exists for every $\alpha>1$.

In $\S 4$ we discuss some properties of $m^{*}(\alpha)$. We show that

$$
m^{*}(\alpha)=d w(\alpha) / d \alpha,
$$

where $w(\alpha)$ is as in (1.6). We also prove that

$$
m^{*}(\alpha) \ll \rho(\alpha),
$$

where $\rho(\alpha)$ is as in (1.5). One can deduce from either (1.9) or (1.10) that as $\alpha \rightarrow \infty$

$$
m^{*}(\alpha) \ll \exp \{-\alpha \log \alpha-\alpha \log \log \alpha+O(\alpha)\} .
$$

We also prove that $m^{*}(\alpha)$ changes sign at least once in every interval of length 2 contained in $[2, \infty)$.

From (1.11) it is clear that (1.8) fails to yield an asymptotic estimate for $M^{*}(x, y)$ if $\alpha$ is large. So in $\$ \S 5$ and 6 we consider the problem of asymptotically estimating $M^{*}(x, y)$ for long ranges of $\alpha$.

In $\S 7$ we use the results of $\S 6$ along with (1.10) to show that

$$
\sup _{\exp \left\{(\log x)^{5 / 8+\varepsilon}\right\} \leqslant y \leqslant x}\left|\frac{M^{*}(x, y)}{\Psi(x, y)}\right| \ll_{\varepsilon}(\log x)^{-1}
$$

holds for every $\varepsilon>0$. This partially sett'es a conjecture due to Erdös that $M^{*}(x, y)$ $=o(\Psi(x, y))$ as $x \rightarrow \infty$, uniformly for $y \geqslant 2$. 
Next, we show in $\S 8$ by the use of $(1.7),(1.8)$ and the results of $\S 4$, that $M^{*}(x, y)$ changes sign quite often if $y$ is not too small in comparison with $x$. On the other hand we showed in [2] that $M(x, y)<0$ for all large $x$ provided

$$
\exp \left\{(\log x)^{5 / 8+\varepsilon}\right\}<y \leqslant x-x^{7 / 12+\varepsilon}
$$

where $\varepsilon>0$ is arbitrary.

Finally we use estimates (1.7), (1.8) and the results of $\S 7$ to show that if $|g| \leqslant 1$, then

$$
\sup _{g}\left|\sum_{2 \leqslant n \leqslant x} \mu(n) g(P(n))\right| \ll \frac{x}{\log x}
$$

and discuss some consequences of (1.12).

Though $M^{*}(x, y)$ has existed implicitly in the literature, not much attention has been paid to it except in the special case $y=x+1$, which is the well-known sum $M(x)=\sum_{1 \leqslant n \leqslant x} \mu(n)$. Various qualitative results have been established for functions that generalize $\Psi(x, y)$ and $\Phi(x, y)$. In most instances these results do not apply to $M^{*}(x, y)$ except in a weak form. The standard reference for such questions is a long paper of Levin and Fainleib [9], which does include a discussion of $M^{*}(x, y)$. But there are some mathematical errors in [9], and their treatment also is extremely complicated. Our emphasis has been on applications and so our results are quite quantitative. Also, many of our results are new.

All the notation introduced so far will be retained. In addition $p$ and $q$ will always denote primes. By $c_{1}, c_{2}, \ldots$ we mean absolute positive constants and implicit constants are absolute unless otherwise indicated. Also for $y \geqslant 1, R(y)$ will denote a monotonic decreasing function of $y$ that satisfies the inequality

$$
R(y) \ll \exp \left\{-c(\log y)^{3 / 5}(\log \log y)^{-1 / 5}\right\} \text { for } y \geqslant 3,
$$

where the constant $c>0$ and the function $R(y)$ are not necessarily the same when used in different contexts. We shall also make frequent use of the following well-known inequalities which follow from the Korobov-Vinogradov zero-free region for the Riemann zeta function (see Chandrasekharan [7, Chapters 2 and 3]):

$$
\begin{gathered}
M(y) \ll y R(y) ; \\
\nu+\sum_{1 \leqslant n \leqslant y} \frac{\mu(n)(\log n)^{\nu}}{n} \ll R(y) \quad \text { for } \nu=0,1,
\end{gathered}
$$

where $(\log 1)^{0}=1$; and

$$
|\pi(y)-\operatorname{li}(y)|<y R(y)(\log y)^{-1},
$$

where $\pi(y)=\sum_{p \leqslant y} 1$ and

$$
\operatorname{li}(y)=\int_{2}^{\max (2, y)} \frac{d t}{\log t} .
$$


2. Estimating $M^{*}(x, y)$ when $\sqrt{x} \leqslant y \leqslant x$. The aim of this section is to prove THEOREM 1 . Let $2 \leqslant \sqrt{x} \leqslant y \leqslant x$. Then

$$
M^{*}(x, y)=\frac{x f(\beta)}{\log y}-\frac{x}{\log ^{2} y}\{1+O(R(\beta))\}+O\left(\frac{x}{\log ^{3} x}\right),
$$

where

$$
f(\beta)=\int_{1}^{\beta} \frac{M(t)}{t^{2}} d t
$$

To prove Theorem 1 we need two lemmas.

LEMMA 1. For $\beta \geqslant 1$ we have $f(\beta) \ll R(\beta)$.

ProOF. From (2.1) we obtain by partial integration

$$
f(\beta)=\left.\frac{-M(t)}{t}\right|_{0+} ^{\beta}+\int_{0+}^{\beta} \frac{d M(t)}{t}=\sum_{1 \leqslant n \leqslant \beta} \frac{\mu(n)}{n}-\frac{M(\beta)}{\beta} .
$$

Lemma 1 follows from (2.2), (1.14) and (1.15).

LEMMA 2. Let

$$
F(t)=\int_{1}^{t} \frac{f(u)}{u} d u-1
$$

Then $F(t) \ll R(t)$ for $t \geqslant 1$.

Proof. Let $N$ be a positive integer. From Lemma 1 we get

$$
\begin{aligned}
1+F(N) & =\int_{1}^{N}\left(\int_{1}^{u} \frac{M(v)}{v^{2}} d v\right) \frac{d u}{u}=\int_{1}^{N} \frac{M(v)}{v^{2}}\left(\int_{v}^{N} \frac{d u}{u}\right) d v \\
& =\int_{1}^{N} \frac{M(v)}{v^{2}}(\log n-\log v) d v \\
& =\log N \cdot f(N)-\int_{1}^{N} \frac{M(v) \log v}{v^{2}} d v \\
& =O(R(N))-\int_{1}^{N} \frac{M(v) \log v}{v^{2}} d v
\end{aligned}
$$

Next,

$$
\begin{aligned}
\int_{1}^{N} \frac{M(v) \log v d v}{v^{2}} & =\sum_{n=1}^{N-1} M(n) \int_{n}^{n+1} \frac{\log t}{t^{2}} d t \\
& =\sum_{n=1}^{N-1} M(n)\{h(n)-h(n+1)\}
\end{aligned}
$$

where

$$
h(n)=\frac{1}{n}+\frac{\log n}{n} .
$$


So from (2.4), (2.5), (1.14) and (1.15) we have

$$
\begin{aligned}
\int_{1}^{N} \frac{M(v) \log v d v}{v^{2}} & =\sum_{n=1}^{N}\{M(n)-M(n-1)\} h(n)+O(R(N)) \\
& =O(R(N))+\sum_{1 \leqslant n \leqslant N} \frac{\mu(n)}{n}+\sum_{1 \leqslant n \leqslant N} \frac{\mu(n) \log n}{n} \\
& =-1+O(R(N)) .
\end{aligned}
$$

So from (2.3) and (2.6) we deduce that

$$
F(N)=O(R(N)) .
$$

Lemma 2 follows from (2.7) on observing that

$$
F(t)-F([t]) \ll t^{-1}
$$

holds for all $t>1$, where [ $t]$ is the largest integer $\leqslant t$.

Proof of Theorem 1 . Let $2 \leqslant \sqrt{x} \leqslant y \leqslant x$. If $1 \leqslant n \leqslant x, p \mid n$ and $p \geqslant y$ then $P(n)=p$. Therefore

$$
M^{*}(x, y)=M(x)-\sum_{1 \leqslant n \leqslant x, P(n) \geqslant y} \mu(n)=M(x)-\sum_{y \leqslant p \leqslant x} \sum_{1 \leqslant n \leqslant x, p \mid n} \mu(n),
$$

and on writing $n=m \cdot p$ we get

$$
M^{*}(x, y)=M(x)+O(\beta)+\sum_{1 \leqslant m \leqslant \beta} \mu(m)\left\{\pi\left(\frac{x}{m}\right)-\pi(y)\right\} .
$$

From (2.8), (1.14) and (1.16) we deduce that

$$
M^{*}(x, y)=O(x R(y))+\Sigma_{1}
$$

where

$$
\Sigma_{1}=\sum_{1 \leqslant m \leqslant \beta} \mu(m)\left\{\operatorname{li}\left(\frac{x}{m}\right)-\operatorname{li}(y)\right\} .
$$

We use (1.17) and rewrite (2.10) as

(2.11) $\Sigma_{1}=\sum_{1 \leqslant m \leqslant \beta} \mu(m) \int_{y}^{x / m} \frac{d t}{\log t}=\int_{y}^{x} \frac{M(x / t) d t}{\log t}=x \int_{1}^{\beta} \frac{M(u) d u}{u^{2} \log (x / u)}$.

If we integrate the last integral in (2.11) by parts, we get

$$
\Sigma_{1}=\frac{x f(\beta)}{\log y}-x \int_{1}^{\beta} \frac{f(u) d u}{u \log ^{2}(x / u)} .
$$

If we also integrate by parts the integral in (2.12), then Lemma 2 shows that

$$
\begin{aligned}
\Sigma_{1} & =\frac{x f(\beta)}{\log y}-\left.\frac{x F(u)}{\log ^{2}(x / u)}\right|_{1} ^{\beta}+2 x \int_{1}^{\beta} \frac{F(u) d u}{u \log ^{3}(x / u)} \\
& =\frac{x f(\beta)}{\log y}-\frac{x}{\log ^{2} x}\{1+O(R(\beta))\}+O\left(\frac{x}{\log ^{3} y}\right) .
\end{aligned}
$$

Theorem 1 follows from (2.9) and (2.13). 
Corollary 1. Let $2 \leqslant \sqrt{x} \leqslant y \leqslant x$. If $\beta$ remains fixed as $x \rightarrow \infty$, then

$$
\lim _{x \rightarrow \infty} \frac{M^{*}(x, y)}{x / \log y}=f(\beta)
$$

for every $\beta \geqslant 1$. If $\alpha$ remains fixed as $x \rightarrow \infty$, then

$$
\lim _{x \rightarrow \infty} \frac{M^{*}(x, y)}{x / \log ^{2} y}=-\frac{1}{\alpha^{2}}
$$

for every $\alpha$ satisfying $1<\alpha \leqslant 2$.

Proof. Theorem 1 trivially implies (2.14). Also (2.15) follows from Theorem 1 and Lemma 1. That proves the Corollary.

3. Estimating $M^{*}(x, y)$ for $y \leqslant \sqrt{x}$. Let

$$
m^{*}(\alpha)= \begin{cases}-\frac{1}{\alpha^{2}} & \text { for } 1<\alpha \leqslant 2, \\ \frac{1}{\alpha^{2}}\left[1+\int_{2}^{\alpha} s m^{*}(s-1) d s\right] & \text { for } \alpha>2 .\end{cases}
$$

In this section we prove the following result:

THEOREM 2. Let $2 \leqslant y \leqslant \sqrt{x}$. Then

$$
M^{*}(x, y)=\frac{x m^{*}(\alpha)}{\log ^{2} y}+O\left(\frac{x R\left(x / y^{2}\right)}{\log ^{2} y}\right)+O\left(\frac{x \cdot \alpha^{2}}{\log ^{3} y}\right)
$$

holds uniformly in $x, y$ and $\alpha$.

For the proof of Theorem 2 and for later use we need

Lemma 3. Let $x, y$ and $T$ satisfy $2 \leqslant y<T \leqslant x$. Then uniformly we have

$$
\sum_{y \leqslant p<T} M^{*}\left(\frac{x}{p}, p\right)-\int_{y}^{T} M^{*}\left(\frac{x}{\xi}, \xi\right) \frac{d \xi}{\log \xi} \ll x R(y) \alpha .
$$

Proof. From (1.1) and (1.16) we get

$$
\begin{aligned}
\left|\sum_{y \leqslant p<T} M^{*}\left(\frac{x}{p}, p\right)-\int_{y}^{T} M^{*}\left(\frac{x}{\xi}, \xi\right) \frac{d \xi}{\log \xi}\right| & \\
& =\left|\sum_{y \leqslant p<T} \sum_{1 \leqslant n \leqslant x / p, P(n)<p} \mu(n)-\int_{y}^{T}\left(\sum_{1 \leqslant n<x / \xi, P(n)<\xi} \mu(n)\right) \frac{d \xi}{\log \xi}\right| \\
& =\mid \sum_{1 \leqslant n \leqslant \beta, P(n)<x / n} \mu(n)\left\{\sum_{\max (P(n), y)<p<\min (x / n, T)} 1\right. \\
& \ll \sum_{1 \leqslant n \leqslant \beta} \frac{x}{n} \frac{R(y)}{\log y} \ll x R(y) \alpha,
\end{aligned}
$$

and that proves Lemma 3. 
Proof of Theorem 2 . We begin by observing that if $2 \leqslant y<y^{h} \leqslant x$, then

$$
M^{*}(x, y)=M^{*}\left(x, y^{h}\right)+\sum_{y \leqslant p<y^{h}} M^{*}\left(\frac{x}{p}, p\right) .
$$

From Lemma 3 and (3.2) we get

$$
M^{*}(x, y)=M^{*}\left(x, y^{h}\right)+\int_{y}^{y^{h}} M^{*}\left(\frac{x}{\xi}, \xi\right) \frac{d \xi}{\log \xi}+O(x \cdot \alpha \cdot R(y)) .
$$

We will deduce Theorem 2 from (3.3) and Theorem 1 by induction on $[\alpha]$.

First assume that $2<\alpha \leqslant 3$. Choose $y^{h}=\sqrt{x}$ in (3.3) and note that $1<$ $\log (x / \xi) / \log \xi \leqslant 2$. So by Theorem 1 we have

$$
\begin{aligned}
\int_{y}^{\sqrt{x}} M^{*}\left(\frac{x}{\xi}, \xi\right) \frac{d \xi}{\log \xi}= & \int_{y}^{\sqrt{x}} \frac{x f\left(x / \xi^{2}\right) d \xi}{\xi \log ^{2} \xi}+\int_{y}^{\sqrt{x}} \frac{x m^{*}(\log x / \log \xi-1)}{\xi \log ^{3} \xi} d \xi \\
& +O\left(\int_{y}^{\sqrt{x}} \frac{x R\left(x / \xi^{2}\right) d \xi}{\xi \log ^{3} \xi}\right)+O\left(\int_{y}^{\sqrt{x}} \frac{x d \xi}{\xi \log ^{4} \xi}\right) \\
= & I_{1}+I_{2}+I_{3}+I_{4} \text { respectively. }
\end{aligned}
$$

The substitution $\xi=x^{1 / s}$ shows that

$$
I_{2}=\frac{x}{\log ^{2} x} \int_{2}^{\alpha} s m^{*}(s-1) d s .
$$

On the other hand the substitution $x / \xi^{2}=v$ shows that

$$
I_{3} \ll \frac{x}{\log ^{3} y} \int_{y}^{\sqrt{x}} \frac{R\left(x / \xi^{2}\right) d \xi}{\xi} \ll \frac{x}{\log ^{3} y} \int_{1}^{x / y^{2}} \frac{R(v) d v}{v} \ll \frac{x}{\log ^{3} y},
$$

while trivially

$$
I_{4} \ll x / \log ^{3} y
$$

The substitution $x / \xi^{2}=v$ also yields

$$
I_{1}=-2 \int_{x / y^{2}}^{1} \frac{x \cdot f(v) d v}{v \log ^{2}(x / v)}=\frac{2 x}{\log ^{2} x} \int_{1}^{x / y^{2}} \frac{f(v)}{v}\left\{1-\frac{\log v}{\log x}\right\}^{-2} d v
$$

In (3.8) we have $0 \leqslant \log v / \log x \leqslant 1 / 3$. So from Lemma 1 , Lemma 2 and (3.8), we get

$$
I_{1}=\frac{2 x}{\log ^{2} x}\left\{1+O\left(R\left(x / y^{2}\right)\right)\right\}+O\left(\frac{x}{\log ^{3} x}\right)
$$

From Theorem 1 and estimates (3.3) through (3.9) we arrive at

$$
\begin{aligned}
M^{*}(x, y)= & \frac{x}{\log ^{2} x}\left\{1+\int_{2}^{\alpha} s m^{*}(s-1) d s\right\} \\
& +o\left(\frac{x R(x / y)^{2}}{\log ^{2} y}\right)+O\left(\frac{x}{\log ^{3} y}\right),
\end{aligned}
$$

when $2<\alpha \leqslant 3$. Theorem 2 for $2<\alpha \leqslant 3$ follows from (3.10) and (3.1). 
For $x \geqslant \xi \geqslant 2$ let $\theta=\log x / \log \xi$. We assume that there is $\phi(\theta)$, a monotonic increasing function of $\theta$, such that

$$
\left|M^{*}(x, \xi)-\frac{x m^{*}(\theta)}{\log ^{2} \xi}\right|<O\left(\frac{x R\left(x / \xi^{2}\right)}{\log ^{2} \xi}\right)+\frac{x \phi(\theta)}{\log ^{3} \xi}
$$

holds for $2 \leqslant \theta \leqslant \alpha-1$. For instance (3.10) shows that (3.11) is valid for $2<\theta \leqslant 3$.

Therefore if $\alpha>3$, we have

$$
\begin{aligned}
\mid \int_{y}^{\sqrt[3]{x}} M^{*}\left(\frac{x}{\xi}, \xi\right) \frac{d \xi}{\log \xi}- & \int_{y}^{\sqrt[3]{x}} \frac{x m^{*}(\log x / \log \xi-1) d \xi}{\xi \log ^{3} \xi} \mid \\
& <O\left(\int_{y}^{\sqrt[3]{x}} \frac{x R\left(x / \xi^{3}\right) d \xi}{\xi \log ^{3} \xi}\right)+\int_{y}^{\sqrt[3]{x}} \frac{x \phi(\theta-1) d \xi}{\xi \log ^{4} \xi} .
\end{aligned}
$$

Similar to (3.5) the substitution $\xi=x^{1 / s}$ shows that

$$
\int_{y}^{\sqrt[3]{x}} \frac{x m^{*}(\log x / \log \xi-1) d \xi}{\xi \log ^{3} \xi}=\frac{x}{\log ^{2} x} \int_{3}^{\alpha} s m^{*}(s-1) d s .
$$

On the other hand we trivially have

$$
\int_{y}^{\sqrt[3]{x}} \frac{x \phi(\theta-1) d \xi}{\xi \log ^{4} \xi}<\frac{x \phi(\alpha-1)}{\log ^{3} y} .
$$

Similar to (3.8) and (3.6) the substitution $x / \xi^{3}=u$ shows that

$$
\int_{y}^{\sqrt[3]{x}} \frac{x R\left(x / \xi^{3}\right) d \xi}{\xi \log ^{3} \xi} \ll \frac{x}{\log ^{3} y} \int_{1}^{x / y^{3}} \frac{R(u) d u}{u} \ll \frac{x}{\log ^{3} y} .
$$

Now choose $y^{h}=\sqrt[3]{x}$. Then from (3.1), (3.3), (3.10) and estimates (3.12) through (3.15), we arrive at

$$
\left|M^{*}(x, y)-\frac{x m^{*}(\alpha)}{\log ^{2} y}\right| \leqslant \frac{c_{1} \alpha x}{\log ^{3} y}+\frac{x \phi(\alpha-1)}{\log ^{3} y} .
$$

We now choose $\phi$ such that it satisfies (3.11) for $2 \leqslant \theta \leqslant 3$ and

$$
c_{1} \alpha+\phi(\alpha-1)<\phi(\alpha) \text {. }
$$

Clearly

$$
\phi(\alpha)=c_{2} \alpha^{2}
$$

satisfies our requirements. Finally, since $\alpha>3$, the term $O\left(x R\left(x / y^{2}\right) / \log ^{2} y\right)$ can be added to (3.16) without any effect. Theorem 2 follows from (3.10), (3.16), (3.17) and (3.18).

COROLlaRY 2. Let $\alpha$ remain fixed as $x \rightarrow \infty$. Then

$$
\lim _{x \rightarrow \infty} \frac{M^{*}(x, y)}{x / \log ^{2} y}=m^{*}(\alpha)
$$

for every $\alpha>1$.

Proof. If $1<\alpha \leqslant 2$, then (3.19) follows from (2.15) and (3.1). If $\alpha>2$, then (3.19) follows from Theorem 2 and (1.13). That proves Corollary 2. 
4. Some properties of $m^{*}(\alpha)$. Here we establish various properties of $m^{*}(\alpha)$ that will be useful in later sections. We begin with

TheOREM 3. (i) For all $\alpha>1, \alpha \neq 2, m^{*}(\alpha)=d w(\alpha) / d \alpha$.

(ii) For $\alpha>1, m^{*}(\alpha) \ll \rho(\alpha)$.

Proof. From (3.1) we get

$$
\alpha m^{*}(\alpha)^{\prime}=m^{*}(\alpha-1)-2 m^{*}(\alpha), \text { for } \alpha>3 .
$$

It is well known (see [4]) that $w(\alpha)$ satisfies

$$
w(\alpha)=\frac{1}{\alpha}+\frac{1}{\alpha} \int_{2}^{\max (2, \alpha)} w(t-1) d t, \text { for } \alpha>1 .
$$

Therefore $w(\alpha)$ satisfies

$$
\alpha w^{\prime}=w(\alpha-1)-w(\alpha), \text { for } \alpha>2 .
$$

If $h(\alpha)=w^{\prime}(\alpha)$, then (4.3) shows that

$$
\alpha h^{\prime}(\alpha)=h(\alpha-1)-2 h(\alpha), \quad \text { for } \alpha>3 .
$$

It is easy to check from (4.2) and (3.1) that $h(\alpha)=m^{*}(\alpha)$ for $1<\alpha \leqslant 3, \alpha \neq 2$. So Theorem 3(i) follows from (4.1) and (4.4), because the functions $h(\alpha)$ and $m^{*}(\alpha)$ are continuous for $\alpha>3$.

Next, from (4.3) and Theorem 3(i) we get

$$
\alpha m^{*}(\alpha)=-\int_{\alpha-1}^{\alpha} m^{*}(t) d t, \text { for } \alpha>3 .
$$

On the other hand it is well known (see [6]) that

$$
\alpha \rho(\alpha)=\int_{\alpha-1}^{\alpha} \rho(t) d t
$$

and that $\rho(\alpha)>0$ for all $\alpha>1$. So define

$$
B=\sup _{1<\alpha \leqslant 3} \frac{\left|m^{*}(\alpha)\right|}{\rho(\alpha)} .
$$

Clearly $B<\infty$. Choose $B^{*}>B$ and let

$$
\alpha_{0}=\inf \left\{u \mid \frac{\left|m^{*}(u)\right|}{\rho(u)} \geqslant B^{*}\right\} .
$$

Then (4.7) shows that $\alpha_{0}>3$. So by (4.5) and (4.6) we have

$$
\left|m^{*}\left(\alpha_{0}\right)\right| \leqslant \frac{1}{\alpha_{0}} \int_{\alpha_{0}-1}^{\alpha_{0}}\left|m^{*}(t)\right| d t<\frac{B^{*}}{\alpha_{0}} \int_{\alpha_{0}-1}^{\alpha_{0}} \rho(t) d t=B^{*} \rho\left(\alpha_{0}\right)
$$

On the other hand from (4.8) and the continuity of $m^{*}(u) / \rho(u)$ for $u>3$, we get

$$
\left|m^{*}\left(\alpha_{0}\right)\right| \geqslant B^{*} \rho\left(\alpha_{0}\right) \text {. }
$$

Estimates (4.9) and (4.10) are contradictory unless

$$
\left\{u \mid \frac{\left|m^{*}(u)\right|}{\rho(u)} \geqslant B^{*}\right\}=\varnothing
$$


for every $B^{*}>B$. Therefore from (4.11) and (4.7) we deduce that

$$
\left|m^{*}(\alpha)\right| \leqslant B \rho(\alpha) \text { for all } \alpha>1,
$$

and that proves Theorem 3(ii).

Corollary 3. As $\alpha \rightarrow \infty$ we have

$$
m^{*}(\alpha) \ll \exp \{-\alpha \log \alpha-\alpha \log \log \alpha+O(\alpha)\} .
$$

Proof. It is well known (see [4] and [5]) that as $\alpha \rightarrow \infty$

$$
w^{\prime}(\alpha) \ll \exp \{-\alpha \log \alpha-\alpha \log \log \alpha+O(\alpha)\}
$$

and

$$
\rho(\alpha)=\exp \{-\alpha \log \alpha-\alpha \log \log \alpha+O(\alpha)\} .
$$

The corlloary follows from Theorem 3(i) and (4.12) or from Theorem 3(ii) and (4.13).

Our next result is

THEOREM 4. The function $m^{*}(\alpha)$ changes sign infinitely often. In fact every interval of length 2 contained in $[2, \infty)$ has a point where $m^{*}(\alpha)$ changes sign.

To prove Theorem 4 we need

LEMMA 4. The function $m^{*}(\alpha)$ has infinitely many zeros. Every open interval of length 1 contained in $[2, \infty)$ contains a zero of $m^{*}(\alpha)$.

Proof. From (4.3) and Theorem 3(i) we have

$$
\alpha m^{*}(\alpha)=\alpha w^{\prime}(\alpha)=w(\alpha-1)-w(\alpha) .
$$

It follows easily from (4.14), that $m^{*}(\alpha)$ cannot be either positive or negative for all large $\alpha$, because otherwise the left and right sides of (4.14) will be of opposite sign. Therefore $m^{*}(\alpha)=0$ for infinitely many $\alpha$.

If $\alpha_{1}>3$ is a zero of $m^{*}(\alpha)$, then from (4.14) and the mean value theorem we see that $m^{*}\left(\alpha_{2}\right)=0$ for some $\alpha_{2}$ satisfying

$$
\alpha_{1}-1<\alpha_{2}<\alpha_{1}
$$

Lemma 4 follows from (4.15).

Proof of Theorem 4. Let $\alpha_{1}>3$ be a zero of $m^{*}(\alpha)$. By (4.5) we have

$$
\int_{\alpha_{1}-1}^{\alpha_{1}} m^{*}(s) d s=0
$$

It follows easily from (3.1) that $m^{*}(\alpha)$ cannot vanish identically in an interval of length 1 contained in $(1, \infty)$. Therefore from (4.16) we deduce that there is a point $\alpha_{2}$ where $m^{*}(\alpha)$ changes sign and

$$
\alpha_{1}-1<\alpha_{2}<\alpha_{1} \text {. }
$$

Theorem 4 follows from Lemma 4 and (4.17).

5. Construction of $A^{*}(x, y)$. From Corollary 3 we see that Theorem 2 becomes inefficient for large values of $\alpha$. We will now construct a function $A^{*}(x, y)$ that approximates $M^{*}(x, y)$ for much longer ranges of $\alpha$. Our method, though similar, is 
more natural compared to de Bruijn's procedure of constructing an approximation to $\Psi(x, y)$ (see [5]).

We want a function $A^{*}(x, y)$ that satisfies a recurrence similar to (3.3). More precisely we want

$$
A^{*}(x, y)=A^{*}\left(x, y^{h}\right)+\int_{y}^{y^{h}} A^{*}\left(\frac{x}{\xi}, \xi\right) \frac{d \xi}{\log \xi}+E(x, y, h),
$$

where $E(x, y, h)$ is small. We can then show from (3.3) and (5.1) by induction on [ $\alpha$, that $A^{*}(x, y)-M^{*}(x, y)$ is small for long ranges of $\alpha$.

We begin by considering a function $A^{*}(x, y)$ given by

$$
A^{*}(x, y)=\frac{x}{\log y} \int_{1}^{\infty} w\left(\frac{\log x-\log t}{\log y}\right) d r(t),
$$

where $w(u)$ is defined to be zero for $u \leqslant 1$ and $r(t)$ to be chosen later.

From (5.1) and (5.2) we get

$$
\begin{gathered}
E(x, y, h)=\int_{1}^{\infty}\left[\frac{x}{\log y} w\left(\frac{\log x-\log t}{\log y}\right)-\frac{x}{\log y^{h}} w\left(\frac{\log x-\log t}{\log y^{h}}\right)\right. \\
\left.-x \int_{y}^{y^{h}} \frac{w((\log (x / \xi)-\log t) / \log \xi)}{\xi \log ^{2} \xi} d \xi\right] d r(t)
\end{gathered}
$$

From (4.2) it follows by a simple change of variables that

$$
\begin{aligned}
& w\left(\frac{\log x-\log t}{\log y}\right) \frac{1}{\log y} \\
& \quad=w\left(\frac{\log x-\log t}{\log y^{h}}\right) \frac{1}{\log y^{h}}+\int_{y}^{y^{h}} w\left(\frac{\log (x / \xi)-\log t}{\log \xi}\right) \frac{d \xi}{\xi \log ^{2} \xi}
\end{aligned}
$$

holds for $1 \leqslant t \leqslant x / y^{h}$. So the integrand in (5.3) vanishes for $t \leqslant x / y^{h}$. Thus if the integrator $r(t)$ is chosen properly, then $E(x, y, h)$ can be kept small.

From (2.9) and (2.10) we know that for $\sqrt{x} \leqslant y \leqslant x$

$$
M^{*}(x, y)-\Sigma_{1} \ll x R(y) .
$$

We combine (2.11) and (4.2) to recognize that

$$
\Sigma_{1}=\frac{x}{\log y} \int_{1}^{\infty} w\left(\frac{\log x-\log t}{\log y}\right) \frac{M(t) d t}{t^{2}} .
$$

We therefore choose $r(t)=f(t)$ and get

$$
d r(t)=M(t) d t / t^{2}
$$

With this choice of $r(t)$ we have

TheOREM 5. Let $2 \leqslant y \leqslant y^{h} \leqslant x$. Then

$$
E(x, y, h) \ll x R\left(x / y^{h}\right)(\log y)^{-1} .
$$


Proof. From (4.12) and (4.2) we deduce that $w(\alpha)=O(1)$. So from (5.3), (5.4), (5.6), (1.13) and (1.14), we get

$$
E(x, y, h) \ll \frac{x}{\log y} \int_{x / y^{h}}^{\infty} \frac{|M(t)| d t}{t^{2}} \ll x R\left(x / y^{h}\right)(\log y)^{-1},
$$

and that proves Theorem 5.

Theorem 5 is useful when $y^{h}=o(x)$. In fact we will use it only when $y^{h} \leqslant \sqrt{x}$.

6. On the difference $M^{*}(x, y)-A^{*}(x, y)$. The aim of this section is to prove

THEOREM 6. For $2 \leqslant y \leqslant x$ we have

$$
M^{*}(x, y)-A^{*}(x, y) \ll x \alpha^{2} R(y) .
$$

Proof. Let

$$
E^{*}(x, y)=M^{*}(x, y)-A^{*}(x, y) \text {. }
$$

We begin by observing that (2.9) and (5.5) imply

$$
E^{*}(x, y) \leqslant c_{3} x R(y)
$$

when $\sqrt{x} \leqslant y \leqslant x$. So (6.2) shows that Theorem 6 is true when $1<\alpha \leqslant 2$.

Next from (6.1), Theorem 5, (5.1) and (3.3), we deduce that

$$
\left|E^{*}(x, y)-E^{*}\left(x, y^{h}\right)-\int_{y}^{y^{h}} E^{*}\left(\frac{x}{\xi}, \xi\right) \frac{d \xi}{\log \xi}\right| \leqslant c_{4} x \alpha R(y),
$$

when $2 \leqslant y \leqslant y^{h} \leqslant \sqrt{x}$. We will deduce Theorem 6 from (6.3) by induction on $[\alpha]$.

Assume that $\sqrt[3]{x} \leqslant y \leqslant \sqrt{x}$. Choose $y^{h}=\sqrt{x}$. For these values of $x, y$ and $h$, we see that $\xi$ in (6.3) satisfies

$$
\sqrt{\frac{x}{\xi}} \leqslant \xi \leqslant x
$$

So from (6.2), (6.3) and (6.4) we get

$$
\left|E^{*}(x, y)\right|<c_{3} x R(y)+\int_{y}^{y^{h}} \frac{c_{3} x R(\xi) d \xi}{\xi \log \xi}+c_{4} x \alpha R(y) \leqslant c_{5} x \alpha R(y)
$$

for $2<\alpha \leqslant 3$.

Let $c_{6}=2 c_{5}$. We will show by induction on $k$ that there is an increasing sequence $a_{j}$, such that if

$$
x^{1 / j} \leqslant y \leqslant x
$$

then

$$
\left|E^{*}(x, y)\right| \leqslant c_{6} a_{j} x R(y) \text {. }
$$

To begin the induction we observe that (6.5) and (6.2) show that (6.6) is true for $j=2$ and $j=3$. Now let $k \geqslant 4$, and let (6.6) be true up to $k-1$. In view of the induction hypothesis it suffices to show (6.6) for

$$
x^{1 / k} \leqslant y \leqslant x^{1 /(k-1)} \text {. }
$$


With $x, y$ as in (6.7), we choose $h=k /(k-1)$ so that $x^{1 /(k-1)} \leqslant y^{h} \leqslant \sqrt{x}$. Also, for these values of $x, y$ and $h$ we see that $\xi$ in (6.3) satisfies

$$
\left(\frac{x}{\xi}\right)^{1 /(k-1)} \leqslant \xi \leqslant \frac{x}{\xi}
$$

So by induction we have

$$
\begin{aligned}
\left|E^{*}(x, y)\right| \leqslant & c_{6} x a_{k-1} R(y) \\
& +c_{6} \int_{y}^{y^{h}} \frac{x R(\xi) a_{k-1} d \xi}{\xi \log \xi}+c_{6} x \alpha R(y) \\
\leqslant & c_{6} x R(y)\left\{a_{k-1}\left(1+\frac{1}{k-1}\right)+k\right\} .
\end{aligned}
$$

We will choose $a_{k}$ such that

$$
a_{k-1} \frac{k}{(k-1)}+k<a_{k}
$$

Clearly $a_{k}=2 k^{2}$ satisfies (6.9).

Finally, for $y \geqslant 2$ there exists a unique $k$ such that (6.7) is true. Also $k \ll \alpha$. Theorem 6 follows from (6.9) and (6.6) by induction on $[\alpha]$.

ReMARKS. From (5.2) we can get a series expansion for $A^{*}(x, y)$ by repeated use of integration by parts. The procedure is similar to (2.12) and (2.13). With $r(t)$ given by (5.6) we get the representation

$$
A^{*}(x, y)=\frac{x f(\beta)}{\log y}+\frac{x}{\log ^{2} y} \int_{1}^{\beta} m^{*}\left(\frac{\log x-\log t}{\log y}\right) \frac{f(t)}{t} d t
$$

The expansion can be continued, but at each step in the integration by parts the proper anti-derivative has to be chosen to get a term of smaller order of magnitude that its predecessor. This requires a little computation like the one carried out in the proof of Lemma 2. The series expansion is finite, but of arbitrary length. Theorem 6 then shows that we have a similar representation for $M^{*}(x, y)$ by adding the term $O\left(x \alpha^{2} R(y)\right)$ to the expansion for $A^{*}(x, y)$. This is useful when $y$ is large, for then $R(y)$ will not make a significant contribution.

7. Partial solution to a problem of Erdös. Motivated by the classical result (1.14), P. Erdös raised the following problem: Determine the range of values of $y$ with respect to $x$, such that

$$
M^{*}(x, y)=o(\Psi(x, y)), \text { as } x \rightarrow \infty .
$$

In fact he conjectured that (7.1) holds uniformly in $y$, for $y \geqslant 2$. We give a partial solution to this problem by proving the following result:

THEOREM 7. Let $\varepsilon>0$ be arbitrarily small. Then

$$
\sup _{\exp \left\{(\log x)^{5 / 8+\varepsilon}\right\} \leqslant y \leqslant x} \frac{\left|M^{*}(x, y)\right|}{\Psi(x, y)} \ll_{\varepsilon} \frac{1}{\log x} .
$$


Proof. From (6.10), Theorem 6, and Theorem 3(ii), we get

$$
\begin{aligned}
M^{*}(x, y) \ll & \frac{x|f(\beta)|}{\log y}+\frac{x}{\log ^{2} y} \int_{1}^{\beta} \rho\left(\frac{\log x-\log t}{\log y}\right) \frac{|f(t)|}{t} d t \\
& +x \log ^{2} x R(y),
\end{aligned}
$$

for $2 \leqslant y \leqslant x$.

It is clear from (1.5) that $\rho$ is a decreasing function. So Lemma 1 shows that if $y \leqslant \sqrt{x}$, then

$$
\begin{aligned}
\frac{x}{\log ^{2} y} \int_{1}^{\beta} \rho\left(\frac{\log x-\log t}{\log y}\right) \frac{|f(t)|}{t} d t & =\frac{x}{\log ^{2} y}\left\{\int_{1}^{\sqrt{y}}+\int_{\sqrt{y}}^{\beta}\right\} \\
& \ll \frac{x \rho(\alpha-1 / 2)}{\log ^{2} y}+\frac{x R(\sqrt{y})}{\log ^{2} y} .
\end{aligned}
$$

In [6] de Bruijn proved that

$$
\rho(\alpha) \sim \frac{1}{\sqrt{2 \pi \alpha}} \exp \left\{-\alpha \xi+\gamma+\int_{0}^{\xi} \frac{e^{s}-1}{s} d s\right\} \quad \text { as } \alpha \rightarrow \infty,
$$

where $\xi$ is the positive solution of

$$
e^{\xi}-1=\alpha \xi
$$

It follows from (7.4) and (7.5) that

$$
\rho\left(\alpha-\frac{1}{2}\right) \ll \rho(\alpha) \sqrt{\alpha \log \alpha} .
$$

Therefore from (7.2), (7.3), (7.6), (1.13), Lemma 1 and (4.13), we deduce that

$$
\left|M^{*}(x, y)\right| \ll_{\varepsilon} \frac{x \rho(\alpha) \sqrt{\alpha \log \alpha}}{\log ^{2} y} \text { for } \exp \left\{(\log x)^{5 / 8+\varepsilon}\right\} \leqslant y \leqslant \sqrt{x} .
$$

On the other hand, from either (7.2), Lemma 1 and (4.13), or from Theorem 1 and Lemma 1, we see that

$$
\sup _{\sqrt{x} \leqslant y \leqslant x}\left|M^{*}(x, y)\right| \ll \frac{x}{\log x} .
$$

From de Bruijn's main result for $\Psi(x, y)$ (see [5], equation (1.3)), (4.13) and (1.13), it follows after a little computation that as $x \rightarrow \infty$, we have

$$
\Psi(x, y) \sim x \rho(\alpha) \text { for } \exp \left\{(\log x)^{5 / 8+\varepsilon}\right\} \leqslant y \leqslant x .
$$

Theorem 7 follows from (7.7), (7.8) and (7.9), because for $x, y$ satisfying the conditions of the theorem, we have $\log y \geqslant(\log x)^{5 / 8}$ and $\alpha \leqslant(\log x)^{3 / 8}$.

REMARKS. If we assume the Riemann Hypothesis, then we can prove by a minor modification of the above argument that

$$
\sup _{\exp \left\{c_{7} \sqrt{\log x} \cdot \log \log x\right\} \leqslant y \leqslant x} \frac{\left|M^{*}(x, y)\right|}{\Psi(x, y)} \ll \frac{1}{\log x} .
$$


It may be felt that it is perhaps more appropriate to compare $M^{*}(x, y)$ with

$$
\Psi^{*}(x, y)=\sum_{1 \leqslant n \leqslant x, P(n)<y} \mu^{2}(n) .
$$

Methods similar to the proof of Theorem 6 show that

$$
\Psi^{*}(x, y) \sim 6 \Psi(x, y) / \pi^{2},
$$

for $x, y$ satisfying the conditions of Theorem 7 . So it does not matter at this stage whether we have $\Psi$ or $\Psi^{*}$.

Based on Theorem 7 and (7.10) we make the following stronger version of Erdös' conjecture:

$$
\sup _{2 \leqslant y \leqslant x} \frac{\left|M^{*}(x, y)\right|}{\Psi(x, y)} \ll \frac{1}{\log x} .
$$

The problem of determining the size of the ratio

$$
r(x, y)=M(x, y) / \Phi(x, y)
$$

is easier. The function $r(x, y) \rightarrow 0$ as $x \rightarrow \infty$ if and only if $\alpha \rightarrow \infty$ (see [2] for details).

8. Positive and negative values of $M^{*}(x, y)$. For large $x$ and $y, M^{*}(x, y)$ changes sign quite often. More precisely we have

THEOREM 8. (i) Let $\alpha_{0} \geqslant 2$. Then there exists $x_{0}\left(\alpha_{0}\right)$, such that, for all $x \geqslant x_{0}\left(\alpha_{0}\right)$, $M^{*}(x, y)$ changes sign at least once as $y$ ranges over the interval $\left(x^{1 /\left(\alpha_{0}+2\right)}, x^{1 / \alpha_{0}}\right)$.

(ii) Let $N(x)$ denote the frequency with which $M^{*}(x, y)$ changes sign for $y \in(\sqrt{x}, x)$. Then $N(x) \rightarrow \infty$ as $x \rightarrow \infty$.

Proof. If $\alpha_{0}<\alpha<\alpha_{0}+2$, then by Theorem 2 we have

$$
M^{*}(x, y)=\frac{x m^{*}(\alpha)}{\log ^{2} y}+O_{\alpha_{0}}\left(\frac{x}{\log ^{3} y}\right) .
$$

Theorem 8(i) follows from (8.1) and Theorem 4.

To prove Theorem 8(ii) it suffices to prove, in view of Theorem 1 , that $f(\beta)$ changes sign infinitely often as $\beta \rightarrow \infty$.

Let $s=\sigma+i$ and $\sigma>1$. Then

$$
\begin{aligned}
\int_{1}^{\infty} x^{-s}\left(\sum_{n \leqslant x} \frac{\mu(n)}{n}\right) d x & =\sum_{n=1}^{\infty} \frac{\mu(n)}{n} \int_{n}^{\infty} \frac{d x}{x^{s}} \\
& =\frac{1}{s-1} \sum_{n=1}^{\infty} \frac{\mu(n)}{n^{s}}=\frac{1}{(s-1) \zeta(s)},
\end{aligned}
$$

where $\zeta(s)$ is the Riemann Zeta function. Similarly

$$
\int_{1}^{\infty} \frac{M(x)}{x^{s+1}} d x=\frac{1}{s \zeta(s)}, \text { for } \sigma>1 .
$$

Therefore by (2.2), (8.2) and (8.3), we have

$$
\int_{1}^{\infty} \frac{f(x) d x}{x^{s}}=\frac{1}{s(s-1) \zeta(s)}, \text { for } \sigma>1 .
$$


The right-hand side of (8.4) remains analytic for $|s-1|<1$. So if $f(x)$ is of the same sign for all large $x$, then by Landau's theorem (see Apostol [3, pp. 237-248]) the left side, and hence the right side, are analytic for $\sigma>0$. This contradicts the fact that $\zeta(s)$ has zeros on the line $\sigma=\frac{1}{2}$. That proves Theorem 8(ii).

The function $M(x, y)$ behaves differently in this respect. I showed in [2] that for each $\varepsilon>0$, there is $x_{1}(\varepsilon)$, such that

$$
M(x, y) \leqslant 0, \quad \text { if } \exp \left\{(\log x)^{5 / 8+\varepsilon}\right\}<y<x-x^{7 / 12+\varepsilon} .
$$

From (8.3) one can deduce by the use of Landau's theorem, the classical result that $M(x)$ changes sign infinitely often as $x \rightarrow \infty$. Though motivated by this result, Theorem 8 is very much different because it describes the frequency of sign changes of $M^{*}(x, y)$ when $x$ is large and fixed, and $y$ alone varies in an interval that depends on $x$.

9. The sum $\Sigma_{2 \leqslant n<x} \mu(n) g(P(n))$. In an earlier paper [1] I observed that any arithmetic function $g$, satisfies

$$
\sum_{d \mid n, d>1} \mu(d) g(p(d))=-g(P(n)),
$$

and discussed some applications of (9.1). One of the applications involved the result

$$
A(x)=\sup _{|g| \leqslant 1}\left|\sum_{2 \leqslant n \leqslant x} \mu(n) g(p(n))\right|=o(x),
$$

and I discuss this very briefly now since it is relevant for our purpose.

Let $((x))=x-[x]$. If $g$ is a bounded function, then from (9.2) and partial summation we get

$$
\sum_{2 \leqslant n \leqslant x} \mu(n) g(p(n))((x / n))=o(x) .
$$

But then by (9.1) we have

$$
\begin{aligned}
\sum_{2 \leqslant n \leqslant x} g(p(n)) \mu(n)\left[\frac{x}{n}\right] & =\sum_{2 \leqslant n \leqslant x} \sum_{d \mid n, d>1} \mu(d) g(p(d)) \\
& =-\sum_{2 \leqslant n \leqslant x} g(P(n)) .
\end{aligned}
$$

From (9.3) and (9.4) we deduce that if $g$ is bounded, then

$$
\lim _{x \rightarrow \infty} \frac{1}{x} \sum_{2 \leqslant n \leqslant x} g(P(n))=c \Leftrightarrow \sum_{n=2}^{\infty} \frac{\mu(n) g(p(n))}{n}=-c .
$$

The equivalence in (9.5) generalizes the well-known result

$$
\sum_{n=1}^{\infty} \frac{\mu(n)}{n}=0
$$

and has an interesting application to the Prime Number Theorem for Arithmetic Progressions. For details see [1]. 
Later, in [2], I employed asymptotic estimates for $M(x, y)$ and proved that $A(x) \sim 2 x / \log x$, as $x \rightarrow \infty$. Our aim now is to use asymptotic estimates for $M^{*}(x, y)$ along with the dual of $(9.1)$, given by

$$
\sum_{d \mid n, d>1} \mu(d) g(P(d))=-g(p(n)) \text { for } n>1,
$$

to estimate

$$
B(x)=\sup _{|g| \leqslant 1}\left|\sum_{2 \leqslant n \leqslant x} \mu(n) g(P(n))\right|,
$$

so that we can obtain an analogue of (9.5). So we now derive

THEOREM 9. For $x \geqslant 2$ we have $B(x) \ll x / \log x$.

Proof. We first recognize that

$$
\sum_{2 \leqslant n \leqslant x} \mu(n) g(P(n))=\sum_{p \leqslant x} \sum_{2 \leqslant n \leqslant x, P(n)=p} \mu(n) g(P(n)),
$$

and on writing $n=m \cdot p$ this is seen to be

$$
=\sum_{p \leqslant x} g(p) \sum_{2 \leqslant n \leqslant x, P(n)=p} \mu(n)=-\sum_{p \leqslant x} g(p) M^{*}\left(\frac{x}{p}, p\right) .
$$

From (9.7) and (9.8) we see that

$$
B(x)=\sum_{2 \leqslant p \leqslant x}\left|M^{*}\left(\frac{x}{p}, p\right)\right|
$$

Next we write

$$
\begin{aligned}
\sum_{2 \leqslant p \leqslant x}\left|M^{*}\left(\frac{x}{p}, p\right)\right| & =\sum_{p \leqslant \exp \left\{(\log x)^{3 / 4}\right\}}+\sum_{\exp \left\{(\log x)^{3 / 4}\right\}<p \leqslant \sqrt{x}}+\sum_{\sqrt{x}<p \leqslant x} \\
& =S_{1}+S_{2}+S_{3} \text { respectively. }
\end{aligned}
$$

From (4.13) and (7.9) we see that

$$
S_{1} \leqslant \sum_{p \leqslant \exp \left\{(\log x)^{3 / 4}\right\}}=\Psi\left(x, \exp \left\{(\log x)^{3 / 4}\right\}\right) \ll \frac{x}{\log x} .
$$

Next from Theorem 7 we get

$$
S_{2} \ll \frac{1}{\log x} \sum_{\exp \left\{(\log x)^{3 / 4}\right\}<p \leqslant \sqrt{x}} \Psi\left(\frac{x}{p}, p\right) \ll \frac{\Psi(x, \sqrt{x})}{\log x} \ll \frac{x}{\log x}
$$

To estimate $S_{3}$ we use the well-known result (see Halberstam-Richert [8, pp. 105-110])

$$
\pi(x)-\pi(x-t) \ll \frac{t}{\log t} \quad \text { for } 1 \leqslant t \leqslant x .
$$


More precisely from (9.13), (1.13) and (1.14), we get

$$
\begin{aligned}
S_{3} & \ll \sum_{\sqrt{x}<p \leqslant x} \frac{x}{p} R\left(\frac{x}{p}\right) \ll \frac{x}{\log x}+\sum_{x^{2 / 3}<p \leqslant x} \frac{x}{p} R\left(\frac{x}{p}\right) \\
& \ll \frac{x}{\log x}+\sum_{1 \leqslant k \leqslant x^{1 / 3}} \sum_{x /(k+1)<p \leqslant x / k} \frac{x}{p} R\left(\frac{x}{p}\right) \\
& \ll \frac{x}{\log x}+\sum_{1 \leqslant k \leqslant x^{1 / 3}} \frac{x R(k)}{k \log x} \ll \frac{x}{\log x} .
\end{aligned}
$$

Theorem 9 follows from estimates (9.9) through (9.14).

From Theorem 9 and (9.7) we obtain by reasoning similar to (9.3) and (9.4), that if $g$ is a bounded function, then

$$
\lim _{x \rightarrow \infty} \frac{1}{x} \sum_{2 \leqslant n \leqslant x} g(p(n))=c^{\prime}
$$

if and only if

$$
\sum_{n=2}^{\infty} \frac{\mu(n) g(P(n))}{n}=-c^{\prime}
$$

There is however an important difference between this equivalence and (9.5). If $g$ is bounded, then in this case there is always a $c^{\prime}$ that satisfies (9.15) and so (9.16) is always convergent. In fact the value of $c^{\prime}$ is given by the convergent infinite series

$$
\sum_{p \leqslant x} \frac{g(p)}{p} \prod_{q<p}\left(1-\frac{1}{q}\right)=c^{\prime}
$$

To realize this we write

$$
\sum_{2 \leqslant n \leqslant x} g(p(n))=\sum_{p(n) \leqslant \log x}+\sum_{p(n)>\log x}=S_{4}+S_{5} .
$$

By the Eratosthenes-Legendre Sieve and Mertens' Theorem (see [8, pp. 30-36]), we have

$$
\begin{aligned}
S_{4} & =\sum_{p \leqslant \log x} g(p) \sum_{1 \leqslant n \leqslant x, p(n)=p} 1 \\
& =\sum_{p \leqslant \log x} g(p) \sum_{1 \leqslant n \leqslant x / p, p(n) \geqslant p} 1 \\
& =\sum_{p \leqslant \log x} g(p)\left\{\frac{x}{p} \prod_{q<p}\left(1-\frac{1}{q}\right)+O\left(2^{\log x}\right)\right\} \\
& =c^{\prime} x+O\left(\frac{x}{\log \log x}\right)
\end{aligned}
$$

where $c^{\prime}$ is given by (9.17). On the other hand the Selberg sieve method [8, p. 105] shows trivially that

$$
S_{5} \ll \sum_{\substack{1 \leqslant n \leqslant x \\ p(n)>\log x}} 1 \ll x \prod_{q \leqslant \log x}\left(1-\frac{1}{q}\right) \ll \frac{x}{\log \log x}
$$

and so (9.15) follows from (9.18), (9.19) and (9.20). 
In other words the infinite series in (9.16) is always convergent, and (9.17) shows that the sum can be calculated if we rearrange by collecting terms with a common largest prime factor and arrange these terms lexicographically. That is

$$
\begin{aligned}
\sum_{n=2}^{\infty} \frac{\mu(n) g(P(n))}{n} & =\sum_{n=2}^{\infty} \frac{\mu(n) g(P(n))}{n} \\
& =\sum_{p} g(p) \sum_{\substack{n=2 \\
P(n)=p}}^{\infty} \frac{\mu(n)}{n}=-\sum_{p} \frac{g(p)}{p} \prod_{q<p}\left(1-\frac{1}{p}\right)=-c^{\prime},
\end{aligned}
$$

where the dash indicates summation over square free numbers. If we collect the terms with a common largest prime factor in (9.16), we have a genuine rearrangement, because there are only a finite number of square free integers with a given largest prime factor. On the other hand, if we collect the terms of the infinite series in (9.5) with a common smallest prime factor, we do not have a rearrangement, because there are infinitely many square free numbers with a given smallest prime factor.

Acknowledgment. I would like to thank Professors P. Erdös, H. L. Montgomery and E. G. Straus for their advice and encouragement, and the referee for his comments and criticism.

\section{REFERENCES}

1. K. Alladi, Duality between prime factors and an application to the prime number theorem for arithmetic progressions, J. Number Theory 9 (1977), 436-451.

2. Asymptotic estimates of sums involving the Moebius function, J. Number Theory (to appear)

3. T. M. Apostol, Introduction to analytic number theory, Springer-Verlag, Berlin and New York, 1976.

4. N. G. de Bruijn, On the number of uncancelled elements in the Sieve of Eratosthenes, Indag. Math. 12 (1950), 247-256.

5. On the number of integers $\leqslant x$ and free of prime factors $>y$, Indag. Math. $13(1951), 50-60$.

6. On the asymptotic behavior of a function occurring in the theory of primes, J. Indian Math. Soc. 15 (1951), 25-32.

7. K. Chandrasekharan, Arithmetical functions, Springer-Verlag, Berlin and New York, 1970.

8. H. R. Halberstam and H.-E. Richert, Sieve methods, Academic Press, New York, 1974.

9. B. V. Levin and A. S. Fainleib, Applications of some integral equations to problems of number theory, Russian Math. Surveys 22 (1967), 119-204.

Department of Mathematics, University of Michigan, Ann Arbor, Michigan 48109

Current address: School of Mathematics, Institute of Advanced Study, Princeton, New Jersey 08540 